\title{
Application of IOT and Data Analytics for Water Management Process to Assist the Public Administration
}

\author{
S.Venkatasubramanain ${ }^{\# 1}$, V.Ranjani ${ }^{\# 2}$, A.T.Barani VijayaKumar ${ }^{\# 3}$, P.B.Arun Prasad ${ }^{\# 4}$, S.Gowthami ${ }^{\# 5}$ \\ Department of Computer Science and Engineering, \\ Saranathan College of Engineering, Tiruchirappalli, TamilNadu, India. \\ ${ }^{1}$ veeyes@saranathan.ac.in \\ 2ranjani-cse@saranathan.ac.in \\ 3barani-cse@saranathan.ac.in \\ 4arunprasad-cse@saranathan.ac.in \\ gowthami-cse@saranathan.ac.in \\ Saranathan College of Engineering, Tiruchirappalli, Tmail Nadu, India.
}

\begin{abstract}
The need to supply water to the growing citizens is in the hands of government bodies. Proper and accurate calculation and monitoring of water consumption is a laborious process. Planning to provide basic amenity is also slightly difficult. In this paper, the authors suggest a method of monitoring the usage of water and disseminate the data in the cloud so that, the administrator can do a simple data analytics on the data for planning and supply of water. The paper makes use of IOT technology to monitor and transmit data, which could be stored in the cloud. The last section of the paper deals with the data analysis. This technique will reduce the time for plan of action by the government bodies to provide water facility to the public. The authors conclude the paper with the estimation of turnaround time for the plan of action when done manually and with the technology.
\end{abstract}

Keywords - IOT, Water management, IOT data analytics, Cloud data, Ultrasonic sensors

\section{INTRODUCTION}

It is estimated that during the year 2025, 1.8 billon people include poor farmers may be affected by the water scarcity [1]. The scarcity of water seems be one of the biggest problem which is hitting all the countries especially the country of India, where the population grows exponentially. The regions suffering from the water scarcity is also increasing [2]. The government bodies such as local administration is taking care of water supply. Overhead tanks are constructed to manage the water distribution and meet peak demand of water.

With the help of Internet of Things, water distribution, management and planning become more easy for the administrators. In our experiment, an ultrasonic water level sensor which can measure the depth up to 7 meters. The reason for using ultrasonic sensors are for the cheap and effective and less risk of contamination.

The section II deals with the existing works in the same area. Section III deals with the design of IOT based system, section IV deals with the experiment and results and section V deals with the conclusion.

\section{RELATED WORK}

To analyze and alert the pipe bursts, Pipenet [3] uses a bluetooth cluster whose head node collects data from other sensors. The cluster head then sends the data over GSM to the server for processing.

TianrongRao , et.all.,[4] uses zig bee as a communication protocol between the sensor devices.

Kudva et al.[5] uses $40 \mathrm{KHz}$ ultrasonic which is reflected from the bottom of the tank. The proposed system works only for a tank of $4 \mathrm{~m}$ depth.

\section{DESIGN OF IOT BASED WATER LEVEL MONITOR SYSTEM}

An Arduino Uno microcontroller is used to control the whole system. It is interfaced with GSM modem, ultrasonic sensor, Ethernet shield [5]. The distance between the ultrasonic sensor and water is measured and the height level of the water is calculated [6]. 


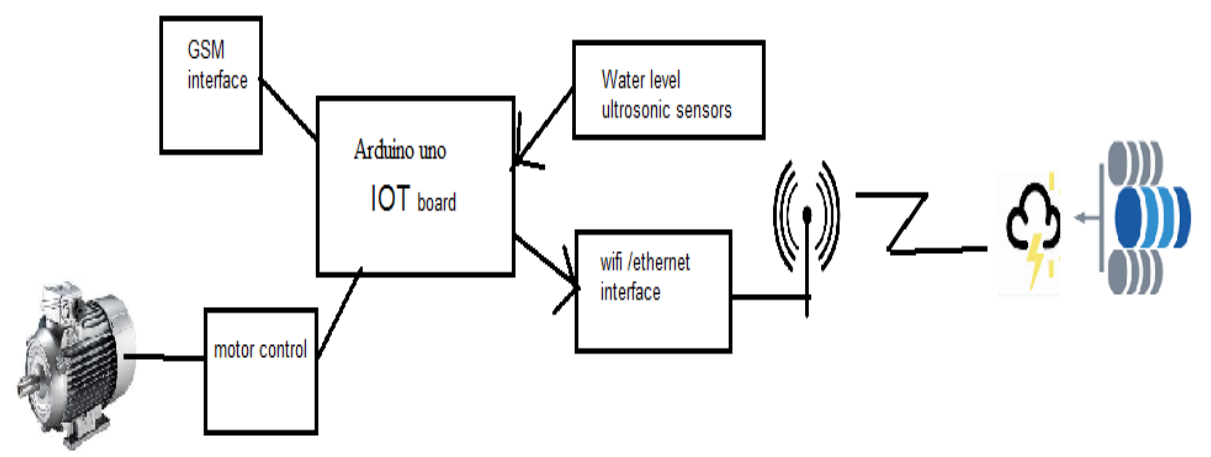

Fig. 1 Water level monitoring and messaging system

1) The Ultrasonic Sensors: In our system, a pair of ultrasonic transducers are used. They are mounted under the lid of the tank or inside ceiling of the water tank with a small gap. The ultrasonic source emits pulse of ultrasonic waves which is reflected at the surface of the water and picked up by the other sensor. More the water in the tank, less time the ultrasonic waves get reflected and less the water level inside the tank more time the waves take to reflect from the surface. The time elapsed between the transmission and reception of the ultrasonic waves is measured. Since the dimension of the water tank is known and each second of delay is calibrated to the volume, the volume of water consumption per time unit is calculated.

2) The Water Level Control : Controlling the level of water is also a subsystem of the paper. The ultrasonic sensors is also used to switch ON/OFF the motor for pumping water. When the water level is low (a threshold values is set) as detected by the sensors, the Uno microcontroller is programmed to signal the motor for pumping. When the water level is high as detected by the sensors, the motor is switched off.

3) The GSM Interface Module: It is used to send text message to the pre-defined numbers whenever information is required to be retrieved.

\section{A. Working of the System}

Two threshold values u1 and v1 represents upper and lower values respectively are set. The values are chosen is such a way that upper threshold value is slightly lesser than the tank capacity and lower threshold values is higher that the tank bottom(i.e. never let water dry inside the tank or level kept higher than the sediment height). The flow chart of the system is presented below:

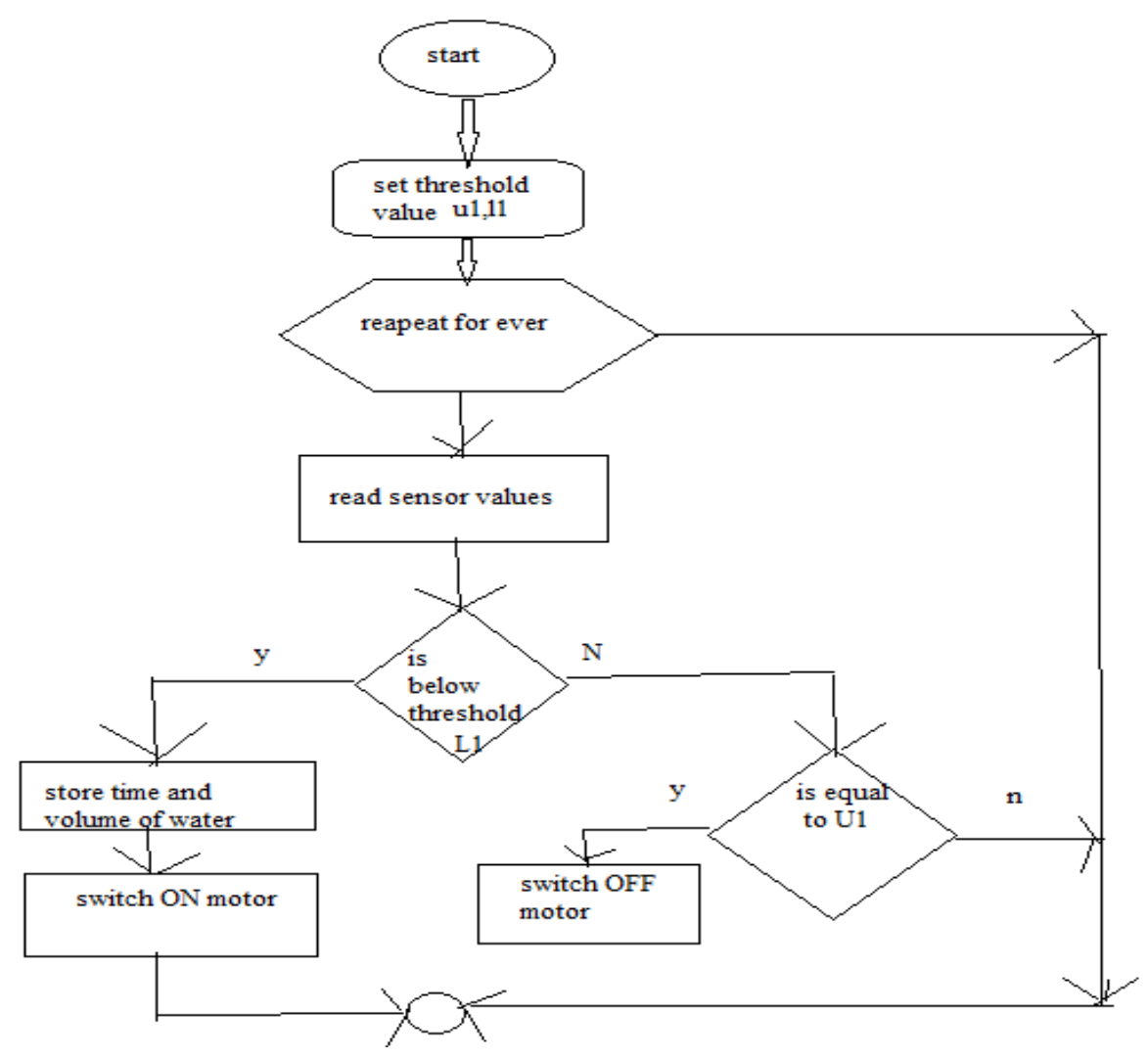

Fig. 2 Flowchart of the System 
The upper and lower threshold values are set and compared from the time delay of ultrasonic waves detected. As already stated more the water, quicker the ultrasonic waves detected and vice versa. The time at which the tank is at the upper threshold value(u1) is measured as t1 and the time at which the lower threshold(l1) detected is t2. The difference between $\mathrm{t} 1$ and $\mathrm{t} 2$ is calculated and rate of consumption of water is also calculated. Theses values are sent to the cloud storage as below. Each tank in each area is programmed an identification code which is sent along with the data(MAC address is mapped).

TABLE I. Data Collected from Various Area Tank

\begin{tabular}{|c|l|l|l|}
\hline Area ID & Population Served & Water Consumed(litres) & Time of Consumption (hours) \\
\hline Area1 & 6000 & 58000 & 23 hours 29 mns \\
\hline Area2 & 9000 & 91000 & 22 hours \\
\hline Area 3 & 3000 & 33000 & 25 hours \\
\hline$\ldots$ & $\ldots$ & $\ldots$ & $\ldots$ \\
\hline$\ldots$ & & $\ldots$ & $\ldots$ \\
\hline
\end{tabular}

The administration department officials can then look into the data and the system present a simple data visualization about the water consumption. The data visualization approach is as shown below in Fig.3 [7].

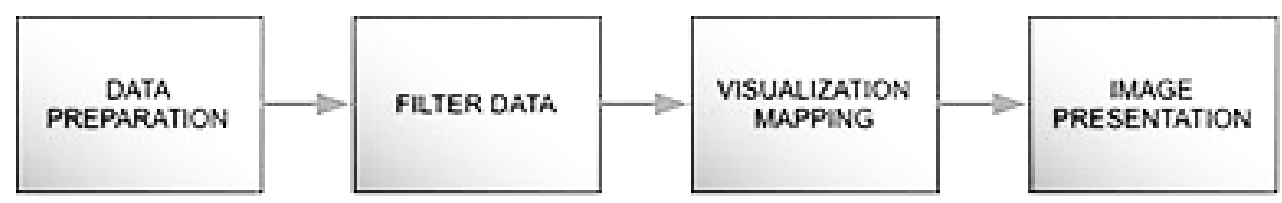

Fig. 3 The data visualization approach
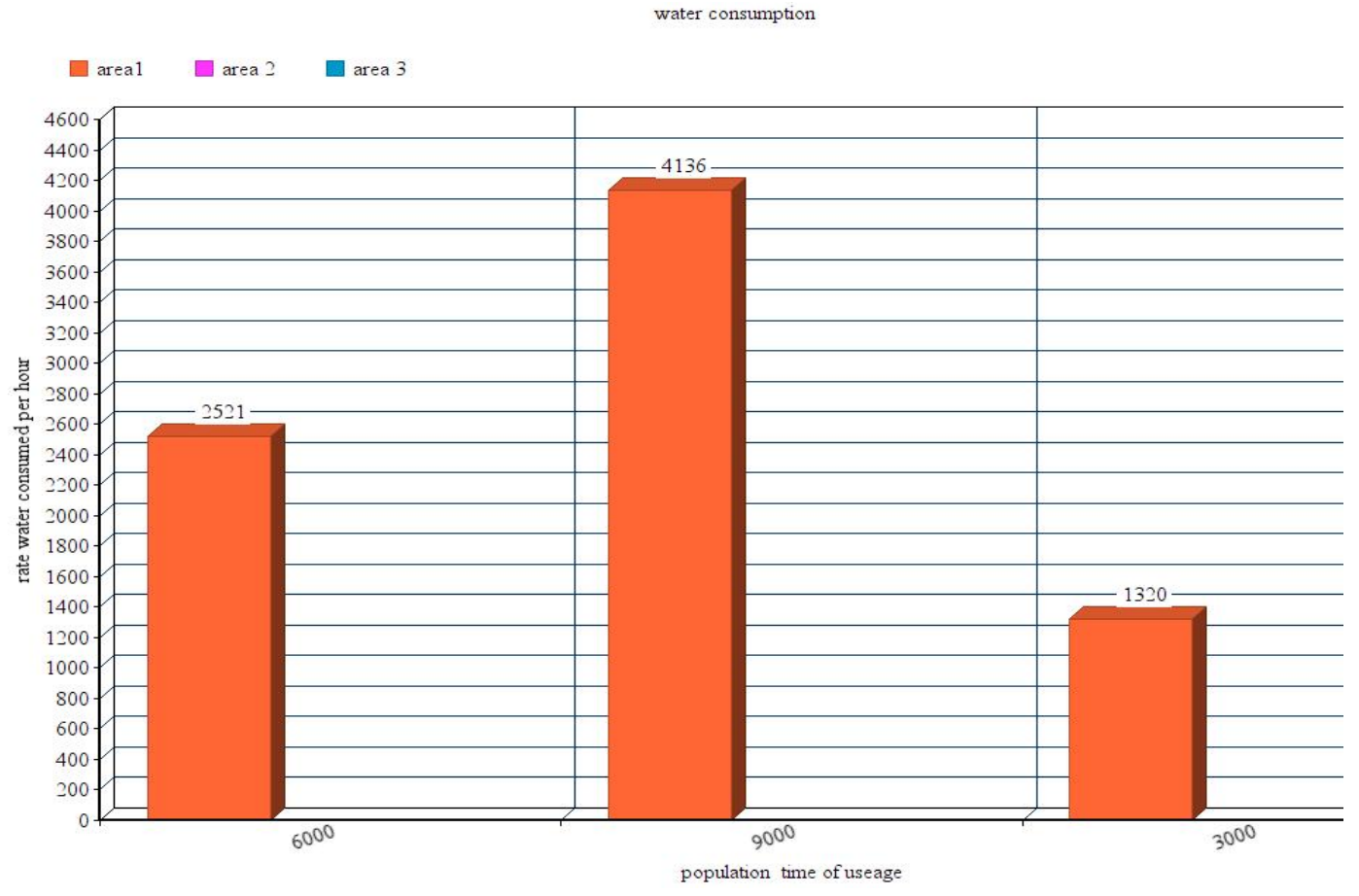

Fig. 4 Simple Data Visualization Presented to the End User 


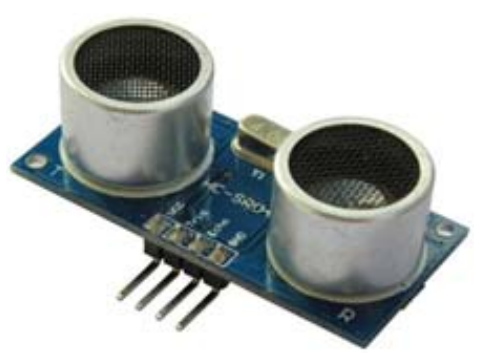

Fig. 5 Ultrosonic Sensor HC SR04

\section{CONCLUSION}

This paper is an outcome of the experimentation with the arduino Uno microcontroller with ultrasonic sensor to measure the water consumption is the overhead tanks of a region. The project could be extended with more data to be presented to the end user such as wastage of water ( usage than usual), special requirements from any area for additional water supply(in case any local festival).

\section{REFERENCES}

[1] UNDP. Human Development Report, Sustaining Human Progress: Reducing Vulnerability and building Resilience. United Nations Development Programme, 2014.

[2] Food and Agriculture Organization of the United Nations. Coping with Water Scarcity: Challenges of the $21^{\text {st }}$ Century. UNFAO, 2007.

[3] Ivan Stoiano. Lama Nachman. Sam madden. Pipenet: A Wireless Sensor Network for pipeline monitoring. In IEEE International Symposium on Information Processing in Sensor Networks, Cambridge, Massachusetts,2007.

[4] TianrongRao, Qiang Ling, Binfeng Yu and HaiboJi, "Estimate the densities of pollutions in water quality monitoring systems based on UV/vis spectrum,” 26th Chinese Control and Decision Conference (CCDC), 2014, pp. 2984-2989.

[5] Vignesh Kudva, et al. Towards a real-time campus-scale water balance monitoring System. In VLSI Design, 2014

[6] Pracet Verma, et al. “Towards IOT based water management system for Campus”, IEEE 2015, 978-4673-6552-9/15.

[7] P. F. Vitiello and R. S. Kalawsky, "Visual analytics: A sensemaking framework for systems thinking in systems engineering," 2012 IEEE International Systems Conference SysCon 2012, pp. 1-6, Mar. 2012 